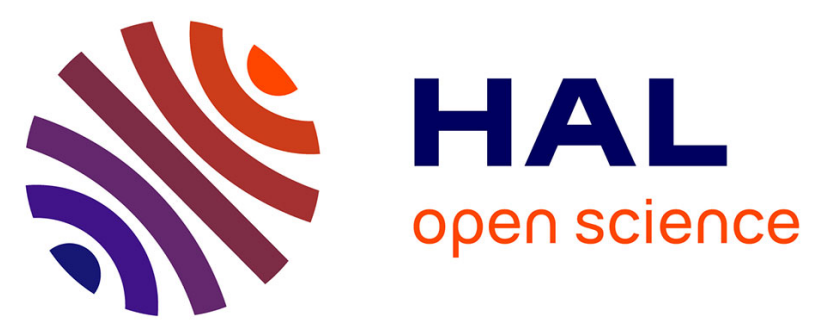

\title{
A two-way coupling CFD method to simulate the dynamics of a wave energy converter
}

Michel Bergmann, Giovanni Bracco, Federico Gallizio, Ermanno Giorcelli, Angelo Iollo, Giuliana Mattiazzo, Maurizio Ponzetta

\section{To cite this version:}

Michel Bergmann, Giovanni Bracco, Federico Gallizio, Ermanno Giorcelli, Angelo Iollo, et al.. A two-way coupling CFD method to simulate the dynamics of a wave energy converter. OCEANS15 MTS/IEEE, May 2015, Genova, Italy. 10.1109/OCEANS-Genova.2015.7271481 hal-01251419

HAL Id: hal-01251419

https://hal.inria.fr/hal-01251419

Submitted on 6 Jan 2016

HAL is a multi-disciplinary open access archive for the deposit and dissemination of scientific research documents, whether they are published or not. The documents may come from teaching and research institutions in France or abroad, or from public or private research centers.
L'archive ouverte pluridisciplinaire HAL, est destinée au dépôt et à la diffusion de documents scientifiques de niveau recherche, publiés ou non, émanant des établissements d'enseignement et de recherche français ou étrangers, des laboratoires publics ou privés. 


\title{
A two-way coupling CFD method to simulate the dynamics of a wave energy converter
}

\author{
Michel Bergmann*, Giovanni Bracco $^{\dagger}$, Federico Gallizio ${ }^{\ddagger}$, Ermanno Giorcelli ${ }^{\dagger}$, \\ Angelo Iollo*, Giuliana Mattiazzo ${ }^{\dagger}$ and Maurizio Ponzetta ${ }^{\S}$ \\ *INRIA Bordeaux Sud-Ouest \\ Bordeaux, France \\ $\dagger$ Dipartimento di Ingegneria Meccanica e Aerospaziale \\ Politecnico di Torino \\ Turin, Italy \\ $\ddagger$ Optimad engineering $\mathrm{Srl}$ \\ Turin, Italy \\ $\S$ Wave for Energy $\mathrm{Srl}$ \\ Turin, Italy
}

\begin{abstract}
The aim of this work is to present a numerical approach to solve accurately the coupled dynamic interaction between a floating body and the incoming wave. This floating body is a Wave Energy Converter (WEC) based on the use of gyroscope in order to extract energy from the slope of the sea waves. The hydrodynamic model is based on a Computational Fluid Dynamic (CFD) approach suited to simulate incompressible viscous flows around an arbitrary moving and morphing body. The mechanical model of the energy converter is given by the conservation laws of the flywheel angular momentum equipped with a control algorithm designed for the power optimization.
\end{abstract}

The interaction of the hull of the energy converter with an incoming wave is computed by switching on the effect of the gyroscope and the mooring and both of them.

\section{INTRODUCTION}

Over the last years in marine industry the CFD has become a diffused numerical tool from the pre-project to the design phase, with the focus of accurate estimation of loads and reliable improvement of the performance. For example some classical marine applications of CFD are the solution of the Navier-Stokes equations for the water region with the aim of evaluating the polar curves of the hull or the performance characterization of the propeller. This kind of evaluation is oneway, because the reaction of the structure to the hydrodynamic forcing is not considered.

A two-way coupled description of the interactions between fluids and structures is not easy by means of classical meshadapted CFD methods. Since the vessel is a buoyant elastic body and the hydrodynamic actions can change the float attitude, a time-comsuming remesh procedure is required at each time step in order to track the fluid/solid interface. The Immersed Boundary (IB) method (see [1] and [2]) is a numerical approach where the complete simulation can be performed on a Cartesian mesh, which is not adapted to the geometrical boundaries, and the interactions between multiphases interfaces are taken into account by means a proper formulation of the fluid equations. Since no re-meshing procedure is needed, a large savings in code complexity and computational costs is yielded. By means of this formulation, some complex geometries with topological change can be easily handled, the multi-physics simulation is more affordable, moving or morphing geometries can be coupled with internal or external flow.

In this work the problem of simulating via CFD the coupled interaction between the incoming sea waves and a floating wave energy converter is addressed. The evolution in time of this interaction is essential because it influences the power extraction from the energy converter. In the following subsection a survey on the Inertial Sea Wave Energy Converter (ISWEC) which is studied in the present work is provided.

The paper is organized as follows. In section II the mechanical model and the control tecnique of ISWEC are presented. In section III the CFD immersed boundary approach is summarized: in particular an outlook on a floating body problem is addressed. In section IV we describe the simulation setup and the combination between the fluid dynamic and the dynamic of the floater. In section $\mathrm{V}$ the results of three preliminary test cases are presented. Some concluding remarks and perspectives are discussed in the section VI.

\section{A. Overview on ISWEC system}

The Inertial Sea Wave Energy Converter (ISWEC) is a device designed to exploit wave energy through the gyroscopic effect of a flywheel (see [3]). All the mechanical parts are enclosed in a sealed monolithic hull that externally appears as a moored boat. A lot of studies and experimental tests have been carried out on this device proving the concept feasibility and estimating its annual energy production (see [4]). The first full scale ISWEC demonstrator with rated power $100 \mathrm{~kW}$ is going to be deployed by Wave for Energy Srl, spin-off of the Politecnico di Torino, at the island of Pantelleria by May 2015.

\section{ISWEC DYNAMICAL MODEL}

As shown in figures 1 and 2, the ISWEC device is composed mainly of a floating body slack moored to the 


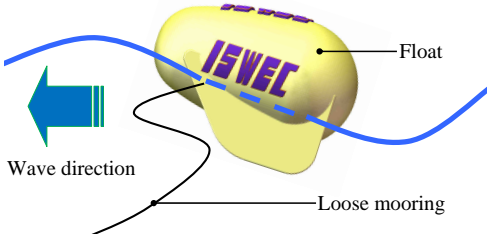

Fig. 1. ISWEC: external appearance (concept).

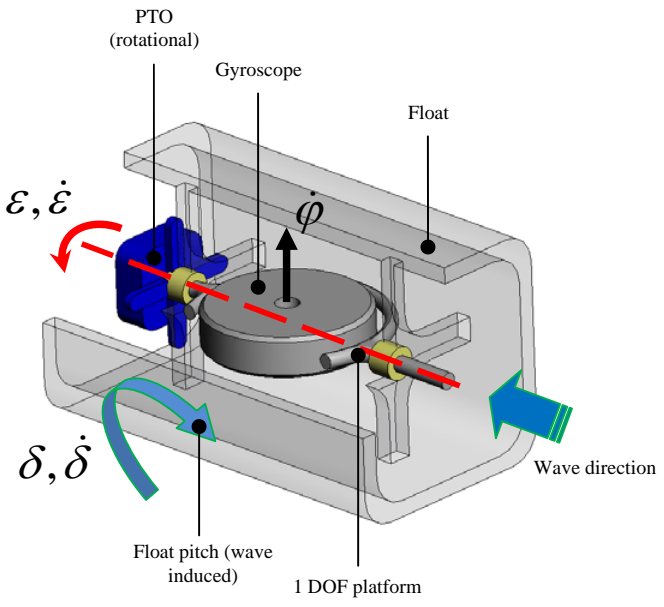

Fig. 2. ISWEC: gyroscopic system.

seabed. The waves tilt the buoy with a rocking motion that is transmitted to the gyroscopic system inside the buoy. The gyroscopic system is composed of a spinning flywheel carried on a platform allowing the flywheel to rotate along the $y_{1}$ axis. As the device works, the gyroscopic effects born from the combination of the flywheel spinning velocity $\dot{\varphi}$ and the wave induced rocking velocity $\dot{\delta}$ create a torque along the $\varepsilon$ coordinate. Using this torque to drive an electrical generator the extraction of energy from the system -and therefore from the waves- is possible.

The reference frames used in the analysis of the system are shown in figure 3. A mobile reference frame $x_{1} y_{1} z_{1}$ is obtained respect to the inertial reference frame $x y z$ with two subsequential rotations $\delta$ and $\varepsilon$.

The mechanical behavior of the system can be easily explained by starting from the initial position in which $\delta=0$ and $\varepsilon=0$, there are no waves and the flywheel rotates around

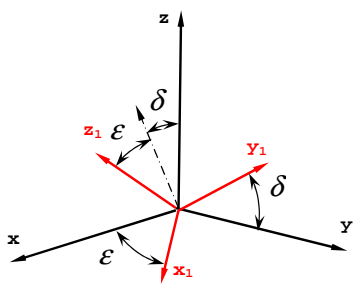

Fig. 3. Reference frames. the axis $z_{1}$ with constant angular velocity $\dot{\varphi}$. As a effect of the first incoming wave, the system is tilted along the pitch direction $\delta$ gaining a certain angular velocity $\dot{\delta}$ along the $x$ axes. The flywheel is so subjected to the two angular velocities $\dot{\varphi}$ and $\dot{\delta}$ and the gyroscopic effects produce a torque on the direction $y_{1}$ that is perpendicular to both the velocities. If the gyroscope is free to rotate along the $\mathrm{y}_{1}$ direction with rotation $\varepsilon$, its behavior is governed only from the inertia and being the system conservative there is no mechanical power available for generation. The extraction of energy from the system can be performed by damping the motion along the $\varepsilon$ coordinate. In this situation the gyroscope acts as a motor on the rotary damper and the energy extracted from the system by the damper is available for power generation. The damper can be for instance an electric generator directly coupled on the $\varepsilon$ shaft. During the evolution of the system -damped or undamped- a gyroscopic torque arises on the buoyant too. In fact the two angular velocities $\dot{\varphi}$ and $\dot{\varepsilon}$ combined together produce a gyroscopic reaction on the buoyant along the $\delta$ coordinate opposing the wave induced pitching motion. And a second reaction torque is similarly induced on the $z_{1}$ axis by the combination of the $\dot{\delta}$ and $\dot{\varepsilon}$ angular velocities. These two reaction torques are to be taken into account when sizing respectively the buoyant and the motor driving the flywheel.

The angular velocity $\vec{\omega}_{1}$ of the mobile reference frame $x_{1} y_{1} z_{1}$ and the flywheel angular velocity $\vec{\omega}_{G}$ are both written with respect to the mobile reference frame - assuming $\vec{i}_{1} \vec{j}_{1} \vec{k}_{1}$ the versors associated to the mobile reference frame $x_{1} y_{1} z_{1}$.

$$
\begin{gathered}
\vec{\omega}_{1}=\dot{\delta} \cos \varepsilon \cdot \vec{i}_{1}+\dot{\varepsilon} \cdot \vec{j}_{1}+\dot{\delta} \sin \varepsilon \cdot \vec{k}_{1} \\
\vec{\omega}_{G}=\dot{\delta} \cos \varepsilon \cdot \vec{i}_{1}+\dot{\varepsilon} \cdot \vec{j}_{1}+(\dot{\delta} \sin \varepsilon+\dot{\varphi}) \cdot \vec{k}_{1} \\
\vec{M}_{e}=\frac{d \vec{K}_{G}}{d t}
\end{gathered}
$$

The (3) expresses the conservation of the angular momentum written with respect to the centre of gravity of the system. The equation describes the rotational equilibrium a mechanical system, asserting that the variation with respect to time of the angular momentum is equal to the applied external torque. In this analysis $\mathbf{J}$ is the moment of inertia of the flywheel around its axis of spinning $z_{1}$ and I represents the two moments of inertia of the flywheel with respect to the axes perpendicular to $z_{1}$.

$$
\vec{K}_{G}=\vec{I} \cdot \vec{\omega}_{G}=I \dot{\delta} \cos \varepsilon \cdot \vec{i}_{1}+I \dot{\varepsilon} \cdot \vec{j}_{1}+J(\dot{\delta} \sin \varepsilon+\dot{\varphi}) \cdot \vec{k}_{1}
$$

Time deriving the angular momentum leads to time derive even the three versors $\vec{i}_{1} \vec{j}_{1} \vec{k}_{1}$ and at the end of all the mathematical passages, the equilibrium of the system is described by the vectorial equation (6).

$$
\begin{gathered}
\frac{d \vec{i}_{1}}{d t}=\vec{\omega}_{1} \times \vec{i}_{1}=\dot{\delta} \sin \varepsilon \cdot \vec{j}_{1}-\dot{\varepsilon} \cdot \vec{k}_{1} \\
\frac{d \vec{j}_{1}}{d t}=\vec{\omega}_{1} \times \vec{j}_{1}=-\dot{\delta} \sin \varepsilon \cdot \vec{i}_{1}+\dot{\delta} \cos \varepsilon \cdot \vec{k}_{1} \\
\frac{d \vec{k}_{1}}{d t}=\vec{\omega}_{1} \times \vec{j}_{1}=\dot{\varepsilon} \cdot \vec{i}_{1}-\dot{\delta} \cos \varepsilon \cdot \vec{j}_{1}
\end{gathered}
$$




$$
\vec{M}_{e}=\left\{\begin{array}{c}
I \ddot{\delta} \cos \varepsilon+(J-2 I) \dot{\varepsilon} \dot{\delta} \sin \varepsilon+J \dot{\varepsilon} \dot{\varphi} \\
I \ddot{\varepsilon}+(I-J) \dot{\delta}^{2} \sin \varepsilon \cos \varepsilon-J \dot{\varphi} \dot{\delta} \cos \varepsilon \\
J(\ddot{\delta} \sin \varepsilon+\dot{\varepsilon} \dot{\delta} \cos \varepsilon+\ddot{\varphi})
\end{array}\right\}
$$

The torque on the PTO $T_{\varepsilon}$ and the torque on the motor driving the flywheel $T_{\varphi}$ are given respectively by the second and the third scalar equation of the (6).

$$
\begin{gathered}
T_{\varepsilon}=I \ddot{\varepsilon}+(I-J) \dot{\delta}^{2} \sin \varepsilon \cos \varepsilon-J \dot{\varphi} \dot{\delta} \cos \varepsilon \\
T_{\varphi}=J(\ddot{\delta} \sin \varepsilon+\dot{\varepsilon} \dot{\delta} \cos \varepsilon+\ddot{\varphi})
\end{gathered}
$$

As the device works, an inertial torque $T_{\delta}$ is discharged from the gyroscopic system to the floating body along the pitching direction $\delta . T_{\delta}$ can be evaluated projecting $\vec{M}_{e}$ along the $x$ direction.

$$
\begin{aligned}
T_{\delta} & =\vec{M}_{e} \cdot \vec{i} \\
& =\vec{M}_{e} \cdot\left(\cos \varepsilon \cdot \vec{i}_{1}+\sin \varepsilon \cdot \vec{k}_{1}\right) \\
& =\left(J \sin ^{2} \varepsilon+I \cos ^{2} \varepsilon\right) \ddot{\delta}+J \ddot{\varphi} \sin \varepsilon+ \\
& J \dot{\varepsilon} \dot{\varphi} \cos \varepsilon+2(J-I) \dot{\delta} \dot{\varepsilon} \sin \varepsilon \cos \varepsilon
\end{aligned}
$$

Being $T_{\delta}$ the main action unloaded from the gyro to the float, in this work, the generalized torque unloaded from the gyro to the load results as follows.

$$
M_{g}=\left\{\begin{array}{c}
0 \\
T_{\delta} \\
0
\end{array}\right\}
$$

In this work the PTO is controlled to behave as a spring damper system, the stiffness component tuning the system on the wave, the damping component absorbing active power.

$$
T_{\varepsilon}=k \varepsilon+c \dot{\varepsilon}
$$

\section{AN ACCURATE IMMERSED BOUNDARY APPROACH FOR FLOATING BODIES}

The flow under consideration involves two interfaces: the interface between two fluids (air and water), $\Gamma_{f}$ and the interface between the body and the fluids, $\Gamma_{s}$. The intersection between $\Gamma_{f}$ and $\Gamma_{s}$ is called the triple line and its denoted by $\Gamma_{f s}$. The computational domain under consideration, $\Omega$ is divided into subdomains $\Omega_{f}$ filled by the fluids and $\Omega_{s}$ for the body. The domain $\Omega_{f}$ is composed by two domains $\Omega_{f}^{+}$and $\Omega_{f}^{-}$for water and air respectively. Let $\psi_{f}$ be a function such that $\psi_{f}=0$ on $\Gamma_{f}, \psi_{f}<0$ in $\Omega_{f}^{-}$and $\psi_{f}>0$ in $\Omega_{f}^{+}$, and let $\psi_{s}$ be a function such that $\psi_{s}=0$ on $\Gamma_{s}, \psi_{s}>0$ in $\Omega_{s}$ and $\psi_{s}<0$ elsewhere. These two functions are called level set functions. The sketch of the configuration is given in figure 4 .

We consider the incompressible Navier-Stokes equations, where $\boldsymbol{u}$ is the velocity field, $p$ is the pressure field, $\mu$ is the

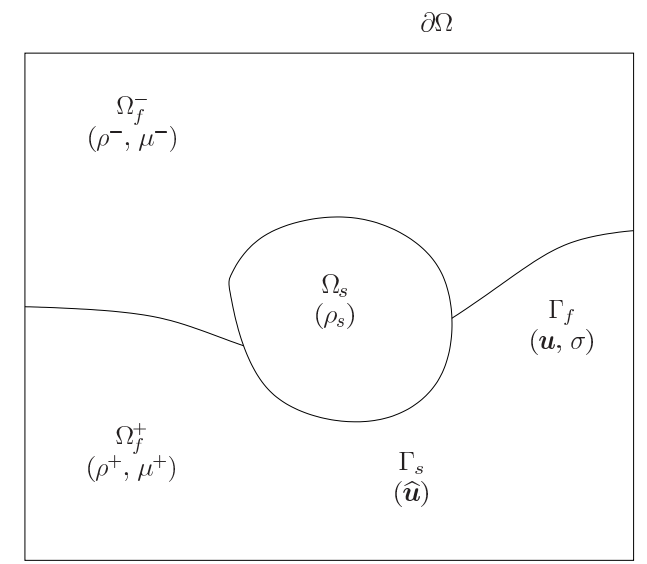

Fig. 4. Sketch of the flow configuration.

dynamic viscosity, $\rho$ is the density, $\boldsymbol{g}$ is the gravity, $\sigma$ is the surface tension between the two fluids, $\boldsymbol{n}$ and $\kappa$ are the normal and curvature of the interface $\Gamma_{f}, \widehat{\boldsymbol{u}}$ is rigid velocity of the body, and $D(\boldsymbol{u})=\frac{\boldsymbol{\nabla} \boldsymbol{u}+\boldsymbol{\nabla}^{T} \boldsymbol{u}}{2}$.

a) In the fluid domain $\Omega_{f}^{+}$et $\Omega_{f}^{-}$:

$$
\begin{gathered}
\rho\left(\psi_{f}\right)\left(\frac{\partial \boldsymbol{u}}{\partial t}+(\boldsymbol{u} \cdot \boldsymbol{\nabla}) \boldsymbol{u}\right)= \\
-\nabla p+\boldsymbol{\nabla} \cdot 2 \mu\left(\psi_{f}\right) D(\boldsymbol{u})+\rho\left(\psi_{f}\right) \boldsymbol{g}, \\
\boldsymbol{\nabla} \cdot \boldsymbol{u}=0
\end{gathered}
$$

with initial and boundary conditions on the external domain boundary $\partial \Omega$.

b) On the interface $\Gamma_{f}$ :

$$
\begin{gathered}
{[\boldsymbol{u}(\boldsymbol{x}, t)]=0,} \\
{[-p I+2 \mu D(\boldsymbol{u})] \cdot \boldsymbol{n}=\sigma \kappa \boldsymbol{n}}
\end{gathered}
$$

c) On the interface $\Gamma_{s}$ :

$$
\boldsymbol{u}(\boldsymbol{x}, t)=\widehat{\boldsymbol{u}}(\boldsymbol{x}, t) .
$$

The viscosity and density are defined as follows:

$$
\begin{aligned}
& \rho\left(\psi_{f}\right)=\rho^{+}+H\left(\psi_{f}\right)\left(\rho^{-}-\rho^{+}\right), \\
& \mu\left(\psi_{f}\right)=\mu^{+}+H\left(\psi_{f}\right)\left(\mu^{-}-\mu^{+}\right),
\end{aligned}
$$

where $H$ denotes the Heaviside function.

The functions $\psi_{f}$ and $\psi_{s}$ are transported with the flow velocity:

$$
\begin{aligned}
& \frac{\partial \psi_{f}}{\partial t}+\boldsymbol{u} \cdot \nabla \psi_{f}=0 \text { in } \Omega . \\
& \frac{\partial \psi_{s}}{\partial t}+\boldsymbol{u} \cdot \boldsymbol{\nabla} \psi_{s}=0 \text { in } \Omega .
\end{aligned}
$$

In practice we chose $\psi_{f}$ and $\psi_{s}$ to be signed distance functions. Since the two last equations do not keep that properties, we have sometimes to reinitialize the level set functions. The displacement of the triple line is computed thanks to the Cox model [9].

We have developped the numerical solver NaSCar based on discretization of the equations on a cartesian mesh. Since 
the interface do not fit the mesh, we impose all the interface boundary condition thanks to some force applied on the momentum equation. We use the CSF (Continuum Surface Force) method [8], [10] to impose the surface tension and used a penalization approach [7], [5] to impose implicitly the boundary condition on the body. We impose a second order penalization developed in [6]. Finally, the system to be solved in the whole domain $\Omega$ is:

$$
\begin{aligned}
\rho\left(\psi_{f}\right)\left(\frac{\partial \boldsymbol{u}}{\partial t}+(\boldsymbol{u} \cdot \nabla) \boldsymbol{u}\right)= & -\nabla p+\nabla \cdot 2 \mu\left(\psi_{f}\right) D(\boldsymbol{u}) \\
& +\rho\left(\psi_{f}\right) \boldsymbol{g}+\sigma \kappa \delta\left(\psi_{f}\right) \boldsymbol{n} \\
& +\frac{\chi}{K}(\widehat{\boldsymbol{u}}-\boldsymbol{u}) . \\
\boldsymbol{\nabla} \cdot \boldsymbol{u} & =0
\end{aligned}
$$

where $\chi$ is the caracteristic function of the body $(\chi=1$ inside the body including the interface $\Gamma_{s}$ and $\chi=0$ outside), $K$ is a penalisation factor, typically $K=10^{-8}$ and the Dirac distribution is regularized:

$$
\delta^{\epsilon}\left(\psi_{f}\right)= \begin{cases}0 & \text { si }\left|\psi_{f}\right|>\epsilon \\ \frac{1}{2 \epsilon}\left(1+\cos \left(\frac{\pi \psi_{f}}{\epsilon}\right)\right) & \text { si }\left|\psi_{f}\right| \leq \epsilon .\end{cases}
$$

The system (12) is discretized in space thanks to second order finite differences (upwind third order for the convertive terms) and equations (11h) and (11i) are discretized using a WENO5 scheme. The discretization in time is perfomed using a predictor-corrector scheme based on the Chorin and Temam scheme [11], [12]. The computation of the body motion will be presented in section IV-B.

\section{Simulation SETUP}

\section{A. Initial and boundary conditions}

System (11) is closed given initial and boundary conditions. This condition tries to mimic a wave propagating in the computational domain staring from a fluid at rest, i.e. $\boldsymbol{u}=0$, with $p$ a hydrostatic pressure computed to balance the gravity force. The domain under consideration is $x \in[-45 \mathrm{~m}, 45 \mathrm{~m}]$, $y \in[-15 m, 15 m]$ and $y \in[-12 m, 18 m]$ where the gravity is $\boldsymbol{g}=-g \boldsymbol{e}_{z}$. The position mass center of the body, $\boldsymbol{x}^{G}$, is initially located at $(x, y, z)=(2 m, 0 m, 0 m)$, i.e. on the water surface so that no buoyancy force is applied.

Periodic boundary conditions are applied on the side boundary (in $x$ and $y$ direction). The bottom is considered as a wall and we thus have $\boldsymbol{u}=\mathbf{0}$. On the top boundary we impose $\frac{\partial u}{\partial z}=\frac{\partial v}{\partial z}=0$ and $w=0$. A snapshot of the initial condition in the computational domain is presented in figure 5 .

\section{B. Integration of the ISWEC model within immersed boundary code}

The motion of the rigid body is computed using Newton's laws from the total forces $\boldsymbol{F}$ and the torques $\mathcal{M}$ exerted by all the external forces onto the body. These forces and torques

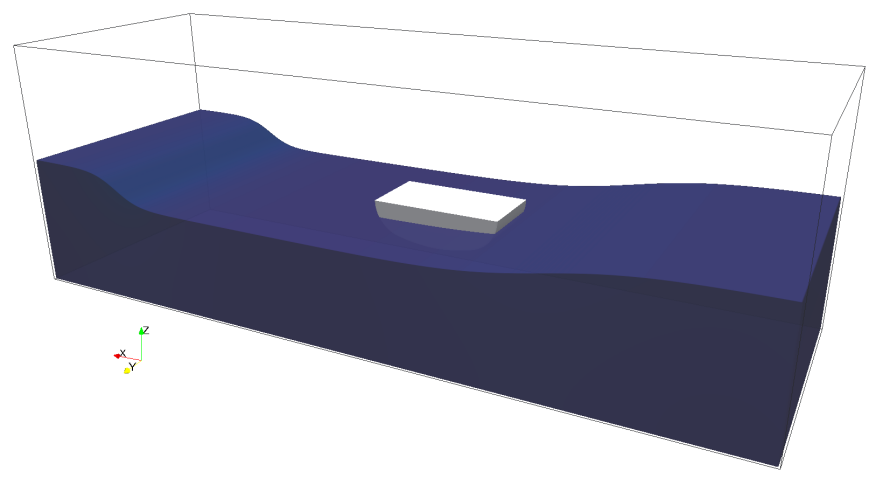

Fig. 5. Snapshot of the initial condition in the computational domain where everything is at rest.

are exerted by (i) the fluid, (ii) a mooring (to avoid a large horizontal displacement) and (iii) the effect of the gyroscope.

The rigid motion is defined by the linear velocity $\bar{u}$ and angular velocity $\Omega$ computed as:

$$
\begin{aligned}
& m \frac{\mathrm{d} \overline{\boldsymbol{u}}}{\mathrm{d} t}=\boldsymbol{F}, \\
& \frac{\mathrm{d} J \boldsymbol{\Omega}}{\mathrm{d} t}=\boldsymbol{M},
\end{aligned}
$$

where $m$ is the total mass of the body and $J$ the inertia matrix.

Let $\mathbb{T}(\boldsymbol{u}, p)=-p \boldsymbol{I}+\frac{1}{R e}\left(\nabla \boldsymbol{u}+\nabla \boldsymbol{u}^{T}\right)$ be the stress tensor, and let $\boldsymbol{n}$ the outward normal unit vector to the body with surface $\partial \Omega_{i}$. The hydrodynamic forces and torques exerted by the fluid onto the body are:

$$
\begin{aligned}
\boldsymbol{F}_{h} & =-\int_{\partial \Omega} \mathbb{T}(\boldsymbol{u}, p) \boldsymbol{n} \mathrm{d} \boldsymbol{x}, \\
\mathcal{M}_{h} & =-\int_{\partial \Omega_{i}} \boldsymbol{r} \wedge \mathbb{T}(\boldsymbol{u}, p) \boldsymbol{n} \mathrm{d} \boldsymbol{x},
\end{aligned}
$$

with $\boldsymbol{r}=\boldsymbol{x}-\boldsymbol{x}^{G}$.

At each time step we computed the fictitious displacement considering only the hydrodynamic forces and torques. This displacement is used to computed the mooring forces $\boldsymbol{F}_{m}$ and torques $\mathcal{M}_{m}$ and the gyroscope forces $\boldsymbol{F}_{g}$ and torques $\mathcal{M}_{g}$. Finally, the total rigid motion is computed using the hydrodynamic, the mooring plus the gyroscope forces and torques.

\section{TEST CASES AND RESUlTS}

Some test cases are considered in order to investigate the correct implementation of the two-way coupled simulations. In particular the following three experiments are performed:

$I$ - hydrodynamic effect: $\boldsymbol{F}=\boldsymbol{F}_{h}$ and $\mathcal{M}=\mathcal{M}_{h}$,

$I I$ - hydrodynamic plus mooring effects $\boldsymbol{F}=\boldsymbol{F}_{h}+\boldsymbol{F}_{h}$ and $\mathcal{M}=\mathcal{M}_{h}+\mathcal{M}_{m}$ and

$I I I$ - hydrodynamic plus mooring plus gyroscope effects $\boldsymbol{F}=\boldsymbol{F}_{h}+\boldsymbol{F}_{h}+\boldsymbol{F}_{g}$ and $\boldsymbol{M}=\mathcal{M}_{h}+\mathcal{M}_{m}+\mathcal{M}_{g}$.

Figures 6,7 and 8 show respectively the motion of the body in the $X$ and $Z$ directions as well as the rotation around 
the $Y$ axis. The effect of the mooring is clearly visible in figure 6 where the mooring action tends to keep the body as close as possible to $\bar{X}=0$. The effect of the gyroscope is also clearly visible in 8 where the rotation of the body is decrease. These phenomena are highlighted in figures 10 and 11 that represent a comparison of snapshots of the simulation for the three test cases at two different simulation time $t=20 \mathrm{~s}$ and $t=22.5 \mathrm{~s}$. Indeed, while the mooring effet is clearly visible (approximatively $2 \mathrm{~m}$ that is quite small is comparison the the $15 \mathrm{~m}$ body length), the effect a the gyroscope is visible.

Figure 9 shows the evolution of the gyroscopic system inside the hull for the test case III. The PTO is regulated to impose a damping coefficient of $30000 \mathrm{Nm} \mathrm{s} / \mathrm{rad}$ and a stiffness of $30000 \mathrm{Nm} / \mathrm{rad}$. The PTO rated torque is $50 \mathrm{kNm}$. The power harvested in such conditions is of $30.65 \mathrm{~kW}$. The torque unloaded from the gyro to the hull is one order of magnitude greater than the torque applied from the PTO: the gyro acts as a gearbox converting relatively small motions of the hull into bigger motions of the PTO shaft and consequently smaller and more manageable torques. Due to the heavy action of the gyro on the hull, the amplitude of rotation $\Theta_{y}$ in test case III is decreased with respect to test I and II.

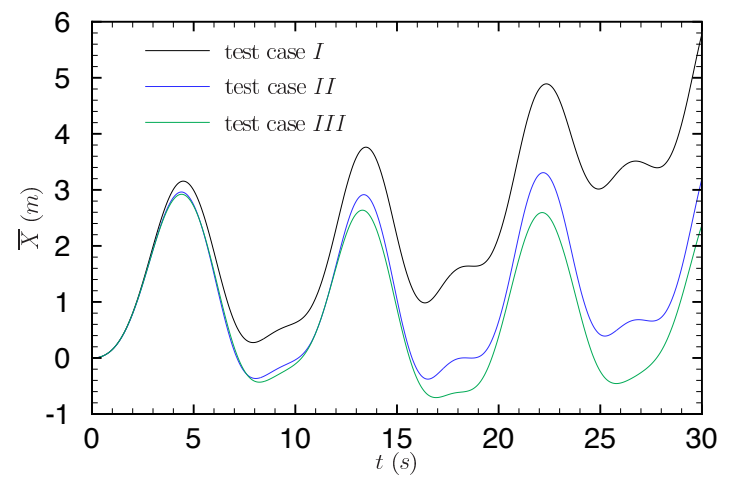

Fig. 6. Temporal evolution of the body displacement in the $X$ direction.

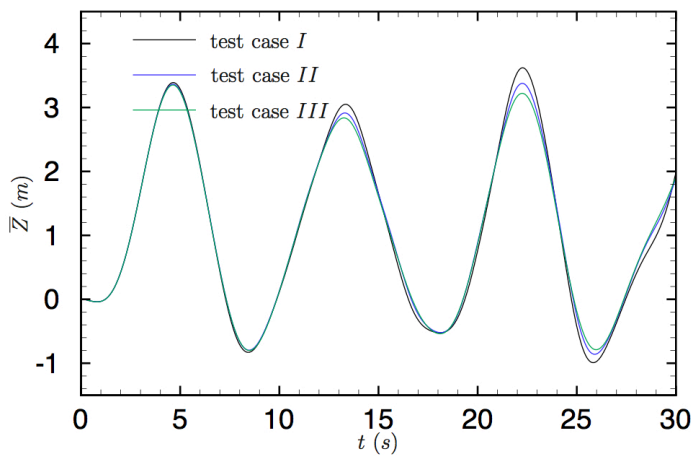

Fig. 7. Temporal evolution of the body displacement in the $Z$ direction.

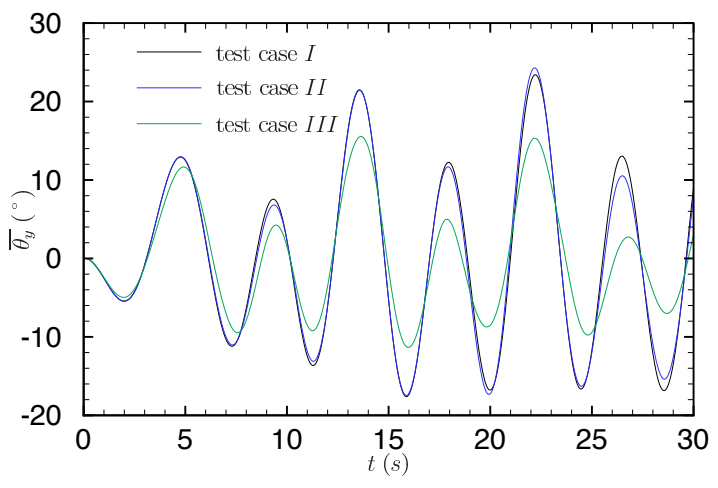

Fig. 8. Temporal evolution of the body rotation $\theta_{y}$.
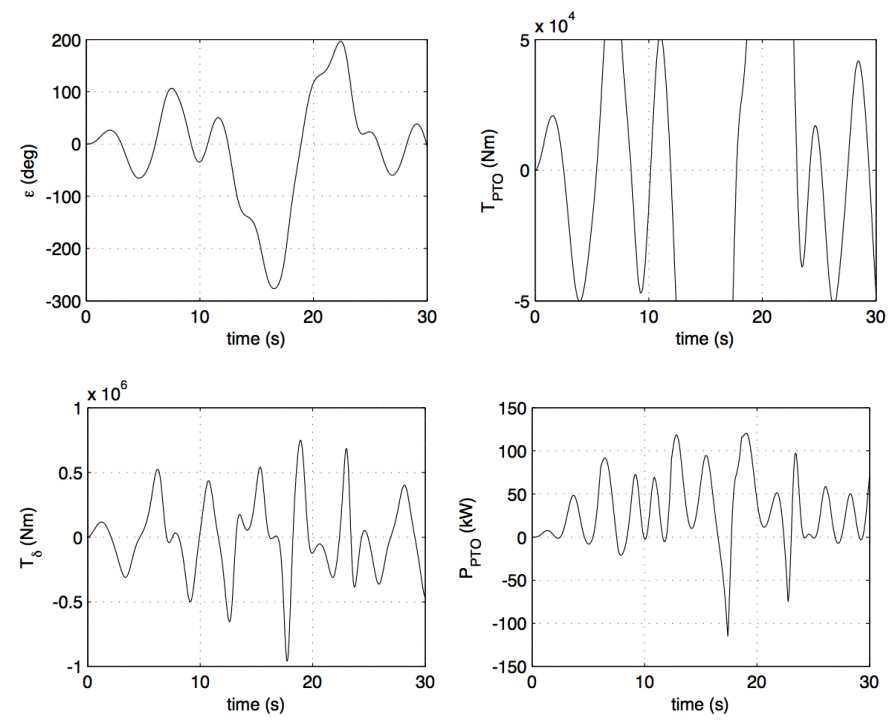

Fig. 9. Time evolution of the characteristic variables of the gyroscopic system.

\section{CONCLUSIONS}

In this paper a CFD technique coupled with a dynamic model of the power generation system were used to simulate the dynamics of an inertial wave energy converter. The CFD method, based on an accurate Immersed Boundary approach, was implemented in order to simulate three test configurations of the wave energy converter for a given wave shape, by switching on the gyroscope and the mooring system. The kinematics of the floater, the torque and the power extracted on the shaft were estimated. Next steps of this work will be the estimation of a sensitivity of the power production by varying the shape and the period of the incoming sea waves.

\section{ACKNOWLEDGMENT}

The research leading to these results has received funding from the European Union Seventh Framework Programme FP7/2007-2013 under agreement no. 309048 (project SiNGULAR). Federico Gallizio would like to thank Dr. Marco Cisternino for the IT advice. 


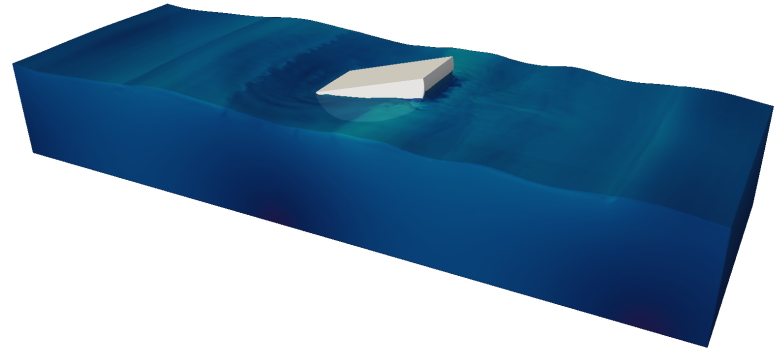

(a) test case $I$.

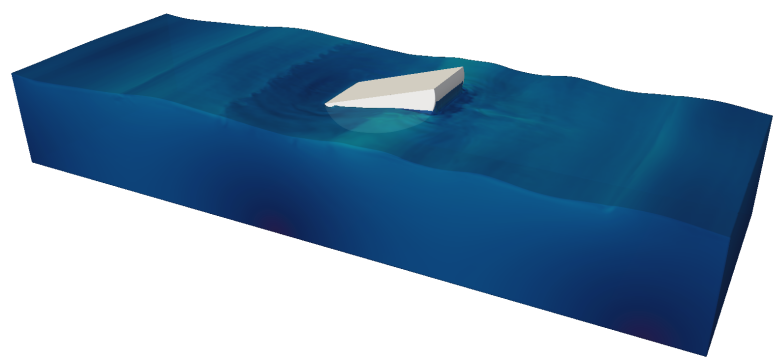

(b) test case $I I$.

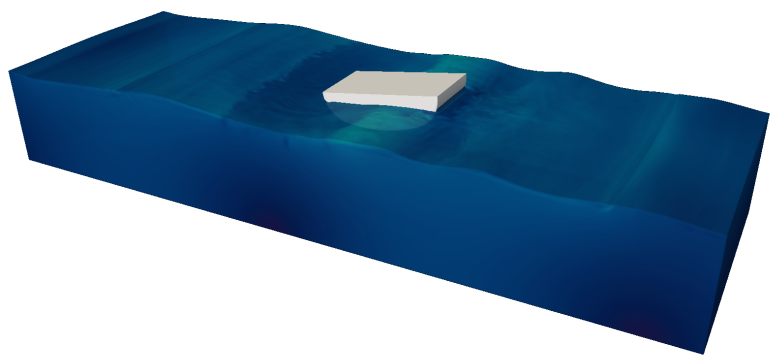

(c) test case $I I I$.

Fig. 10. Snapshots of the simulation at $t=20 \mathrm{~s}$

\section{REFERENCES}

[1] C. Peskin. Flow patterns around the heart valves. Journal of Computational Physics, 10:252-271, 1972.

[2] R. Mittal and G. Iaccarino. Immersed Boundary Methods. Annual Review of Fluid Mechanics, 37:239-261, 2005.

[3] G. Bracco, E. Giorcelli, G. Mattiazzo, M. Pastorelli and J. Taylor. ISWEC: design of a prototype model with gyroscope. IEEE Conference Proceedings, ICCEP, Capri, Italy, 2009.

[4] G. Bracco, E. Giorcelli, and G. Mattiazzo. ISWEC: A gyroscopic mechanism for wave power exploitation. Mechanism and Machine Theory, 46:1411-1424, 2011.

[5] M. Bergmann and A. Iollo, Modeling and simulation of fish-like swimming. Journal of Computational Physics, 230:329-348, 2011.

[6] M. Bergmann, J. Hovnanian and A. Iollo, An accurate Cartesian method for incompressible flows with moving boundaries. Communications in Computational Physics, 15:1266-1290, 2014.

[7] P. Angot, C.H. Bruneau et P. Fabrie : A penalization method to take into account obstacles in a incompressible flow. Num. Math., 81(4):497-520, 1999.

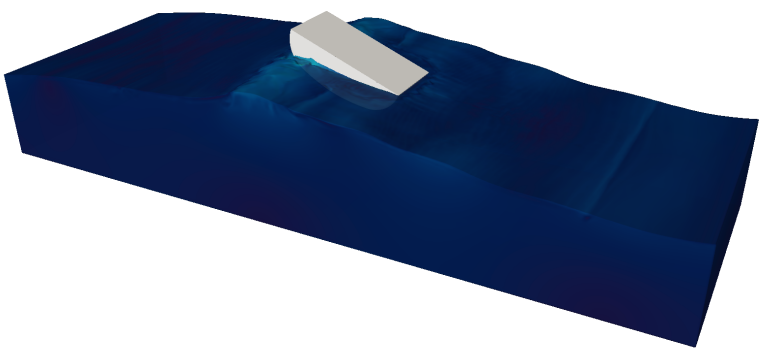

(a) test case $I$.

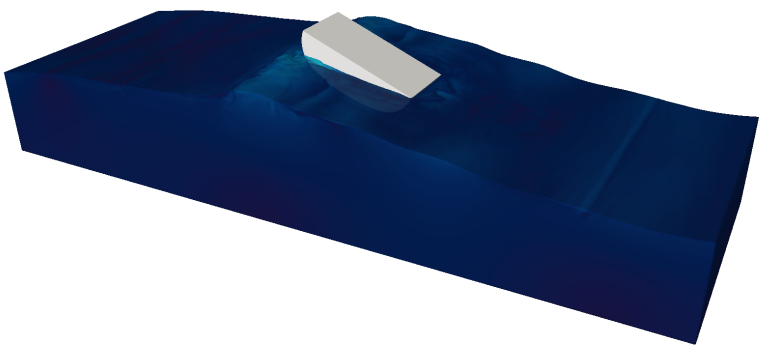

(b) tests case $I I$.

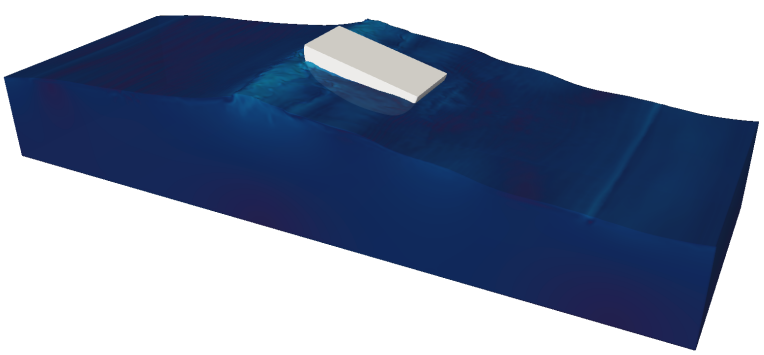

(c) test case $I I I$.

Fig. 11. Snapshots of the simulation at $t=22.5 \mathrm{~s}$.

[8] J.U Brackbill, D.B Kothe et C Zemach : A continuum method for modeling surface tension. Journal of Computational Physics, 100(2):335 - 354, 1992

[9] R.G. Cox : The dynamics of the spreading of liquids on a solid surface. Part 1. Viscous flow. Journal of Fluid Mechanics, 168:169-194, 1986.

[10] M. Sussman, P. Smereka and S. Osher : A level set approach for computing solutions to incompressible two-phase flow. Journal of Computational Physics, 114(1):146 - 159, 1994.

[11] A.J. Chorin : Numerical solution of the Navier-Stokes equations. Math. Comp., 22:745-762, 1968.

[12] R. Temam : Sur l'approximation de la solution des equations de NavierStokes par la methode des pas fractionnaires ii. Archiv. Rat. Mech. Anal., $32: 377-385,1969$ 